\title{
Viscoelastic Properties and Morphology of Mumio-based Medicated Hydrogels
}

\author{
Oyunchimeg Zandraa ${ }^{\mathrm{a}}$, Lenka Jelínková ${ }^{\mathrm{b}}$, Niladri Roy ${ }^{\mathrm{c}}$, Tomáš Sáha ${ }^{\mathrm{c}}$, \\ Takeshi Kitano ${ }^{\mathrm{b}}$ and Nabanita Saha ${ }^{\mathrm{b}}$ \\ ${ }^{a}$ Medical Materials Research Centre, University Institute, Tomas Bata University in Zlin, \\ Nad Ovčirnou 3685,760 01 Zlin, Czech Republic \\ ${ }^{b}$ Centre of Polymer Systems, Polymer Centre, Tomas Bata University in Zlin, \\ nam. T. G. Masaryka 5555, 76001 Zlin, Czech Republic \\ ${ }^{c}$ Centre of Polymer Systems, Tomas Bata University in Zlin, \\ nam. T. G. Masaryka 5555, 76001 Zlin, Czech Republic
}

\begin{abstract}
Novel medicated hydrogels were prepared (by moist heat treatment) with PVA, agar, mumio, mare's milk (MM), seabuckthorn oil (SB oil) and salicylic acid (SA) for wound dressing/healing application. Scanning electron micrographs (SEM) show highly porous structure of these hydrogels. The swelling behaviour of the hydrogels in physiological solution displays remarkable liquid absorption property. The knowledge obtained from rheological investigations of these=systems may be highly useful for the characterization of the newly developed topical formulations. In the present study, an oscillation frequency sweep test was used for the evaluation of storage modulus $\left(G^{\prime}\right)$, loss modulus $\left(G^{\prime}\right)$, and complex viscosity $\left(\eta^{*}\right)$ of five different formulations, over an angular frequency range from 0.1 to $100 \mathrm{rad} . \mathrm{s}^{-1}$. The influence of healing agents and swelling effect on the rheological properties of mumio-based medicated hydrogels was investigated to judge its application on uneven surface of body.
\end{abstract}

Keywords: Medicated hydrogel, Polyvinyl alcohol, Agar, Mumio, Swelling, Viscoelasticity, Morphology.

PACS: 47.50.-d, 47.50.Ef, 47.57.Qk, 83.60.Bc

\section{INTRODUCTION}

Hydrogels are an interesting class of polymeric materials that can retain large amounts of water without dissolving. The properties of these materials depend on their building blocks of crosslinking network and the preparation procedures [1]. Hydrogels have various applications: electrophoresis, water purification, artificial vegetation, oil spill removal and in health care purposes like transdermal drug delivery, wound dressing, wound healing, contact lens, tissue regeneration, implant etc. Recently, hydrogels for healthcare application has been taken a great interest because of its unique features i.e. absorptivity, non adherence, holding a large quantity of water, barrier against microbial penetration, painless removal after use and so on. Beside biocompatibility, material's biodegradability is an important factor because of the renewed attention towards environmental protection issues [2]. Thus, concentration has been given on natural, biodegradable and biocompatible polymers during material selection for hydrogel preparation.

Novel Trends in Rheology IV

AIP Conf. Proc. 1375, 261-271 (2011); doi: 10.1063/1.3604487

(C) 2011 American Institute of Physics 978-0-7354-0935-4/\$30.00 
Polyvinyl alcohol (PVA) hydrogels or agar hydrogels have received increasing attention in biomedical and biochemical applications $[3,4]$ because of their nontoxicity and biocompatibility. However, the use of PVA hydrogels is limited due to low mechanical strength and poor thermal stability [5-7]. Polymer blending is a useful method of improving or modifying the physicochemical properties of polymer materials. Blends between synthetic polymers and biopolymers are of particular significance to current hydrogel research. Agar is known to melt on heating and reset on cooling [6]. This cycle can be repeated for an indefinite number of times without compromising the mechanical properties of the gel. Thus, these gelling characteristics of agar could be used to achieve biocompatible but stronger mechanical property oriented hydrogel dressings and till now only two paper reported about PVA-agar hydrogels $[6,7]$.

Mumio, which is a natural medicine, has a broad spectrum of medicinal value created by nature itself, and has no side effect on human body. It is a mixture of organic and inorganic matters, brown to black, sticky and tenacious material with a shiny and polished surface, easily soluble in water. It contains a unique combination of organic acids, proteins and ferments, which have many medical benefits including immunity enhancement, anti-infection and anti-bacterial properties, blood cell regeneration etc [8].

Though, some information available about PVA-agar hydrogels [6, 7] but no information has been found concerning the use of mumio in hydrogel dressings. 'Mumio gel' for rheumatoid arthritis has already been developed in our laboratory which has no harmful side effect on cell as well as human body. Moreover, there is no report about 'Mumio hydrogel' yet which motivated us for the development of some new medicinal value added hydrogels using mumio and other ingredients for biomedical applications.

Till today, not much information is available regarding study of viscoelastic behaviour of the wound dressing hydrogels [9]. But it is earnestly important according to the application point of view. Whenever hydrogels are applied on body surface (skin), they experience some strain/deformation. To withstand with such kind of external influences, hydrogels must be flexible in nature and the extent of flexibility can be determined by their viscoelastic properties. The viscoelastic properties of hydrogels correlate strongly with their microstructures and could provide useful information for modulating their performance characteristics [10]. The viscoelastic behaviour of gels is dependent on several factors, like composition and concentration of the dispersed materials and the lag time between preparation and measurement [11]. Rheological properties also influence the drug release characteristics of the polymer solutions and hydrogels [12]. When some strain applied on the hydrogel during application some morphological deformation may occur within the hydrogel. Thus, it is essential to investigate the occurrence of morphological changes within hydrogels before and after rheological measurement.

This paper reports about the preparation of mumio-based medicated hydrogels, their composition dependent physical appearance, moisture (water) content, viscoelastic properties under oscillatory flow and morphology. 


\section{EXPERIMENTAL}

\section{Materials}

Partially hydrolyzed polyvinyl alcohol 8-88 (PVA, hydrolysis degree 86.7-88.7 mol. $\%, M_{\mathrm{w}}=67,000 \mathrm{~g} \cdot \mathrm{mol}^{-1}$, polymerization degree 1,400$)$ and natural polysaccharide agar were supplied by Fluka, Switzerland. Mumio and mare's milk (MM) were obtained from Monenzym Co., Ltd. Mongolia. Seabuckthorn oil (SB oil) was purchased from Biomedica spol. s r.o., Czech Republic, salicylic acid (SA) from Ing. Petr Lukeš, Czech Republic. All ingredients were used without any further purification. Distilled water was used for the hydrogel preparation.

In mumio-based medicated hydrogel, PVA functions as a base polymer, agar performs as gelling agent as well as base polymer; mumio, SB oil, MM and SA perform as wound healing agents, respectively.

\section{Hydrogel Preparation}

The mumio based medicated hydrogels were prepared by moist heat treatment $[9,13]$ using aqueous solution of PVA, agar, mumio and MM or SB oil or SA. Five different polymeric solutions were prepared by varying the healing ingredients and keeping the amount of PVA and agar components constant. The compositions of each component in the hydrogels are shown in Table 1.

TABLE 1. Composition of mumio-based medicated hydrogel (dry weight except SB oil) in \%.

\begin{tabular}{lcccccc}
\hline Sample & PVA & Agar & Mumio & SB Oil & MM & SA \\
\hline PVA-agar (control) & 2 & 2 & 0 & 0 & 0 & 0 \\
PVA-agar-mumio & 2 & 2 & 1 & 0 & 0 & 0 \\
PVA-agar-mumio-SB Oil & 2 & 2 & 1 & 1 & 0 & 0 \\
PVA-agar-mumio-MM & 2 & 2 & 1 & 0 & 1 & 0 \\
PVA-agar-mumio-SA & 2 & 2 & 1 & 0 & 0 & 1 \\
\hline
\end{tabular}

The polymer solutions (PVA-agar) were prepared in sealed bottles by heat treatment at the temperature of $120^{\circ} \mathrm{C}$ for 20 minutes. After cooling down to the temperature of $50^{\circ} \mathrm{C}$ healing agents were added according to the Table 1 and thoroughly mixed to get a homogeneous solution. Then, the liquids $(20 \mathrm{ml}$ each $)$ were poured into Petri dishes $(80 \mathrm{~mm}$ diameter) following the solution casting method and allowed to cool at room temperature $\left(23^{\circ} \mathrm{C}\right)$. Hydrogel samples were prepared in a UV sterilized laminar flow box under aseptic conditions. Finally, smooth, transparent/brown, round shaped hydrogels were obtained.

\section{Moisture Content Measurement}

Moisture content of the hydrogels was measured by using Eq. 1 [14], in which: $M_{\mathrm{n}}$ is the moisture content (\%) of material $n, W_{\mathrm{w}}$ is the wet weight of the sample, and $W_{\mathrm{d}}$ is the weight of the sample after drying. The hydrogel samples were left to dry in 
an air box at room temperature $\left(22-23^{\circ} \mathrm{C}\right)$ till they reached a constant weight. Three replicates were taken, and the average value of moisture content was determined

$$
M_{n}(\%)=\left(\frac{W_{w}-W_{d}}{W_{w}}\right) \times 100 .
$$

\section{Morphology}

Scanning electron microscopy (SEM) analysis was carried out on VEGA II LMU (TESCAN) operating in the high-vacuum/secondary electron imaging mode at an accelerating voltage of 5-20 kV. The freeze-dried samples were sputter coated with a thin layer of palladium/gold alloy to improve the surface conductivity and tilted $30^{\circ}$ for better observation. The images were taken at the magnification of $100 \mathrm{x}-2 \mathrm{kx}$.

\section{Swelling Studies}

The degree of swelling can be described as time dependent water absorptivity of the hydrogel. The dry films were obtained by drying the hydrogels at room temperature $\left(22-23^{\circ} \mathrm{C}\right)$ until they reached constant weight. Small pieces $\left(1 \mathrm{~cm}^{2}\right)$ from the dry films were weighed and immersed in physiological solution and then in an oven leaved to swell at $37^{\circ} \mathrm{C}$ temperature until $330 \mathrm{~min}$. After the specified time intervals $(30 \mathrm{~min})$, the surface water on the swollen gels was wiped off with tissue paper, and the wet weight of specimens was determined at room temperature. The samples were re-submerged in physiological solution and replaced again at $37^{\circ} \mathrm{C}$.

The degree of swelling corresponds to the water absorptivity of the material, which is defined by Eq. 2 [10], where $W_{\mathrm{s}}$ and $W_{\mathrm{d}}$ are weights of swollen gel and dried gel, respectively

$$
\text { Water absorptivity }(\%)=\left(\frac{W_{s}-W_{d}}{W_{d}}\right) \times 100 \text {. }
$$

\section{Viscoelastic Measurement}

The viscoelastic behaviour of hydrogels was investigated by using a parallel plate rheometer (ARES; Rheometrics Scientific, USA) testing machine with an "RSI Orchestrator" software package. A 25-mm diameter parallel plate measuring geometry with a gap of about $2-3 \mathrm{~mm}$ was used, employed at small strain amplitude (1\%) to maintain the measurements within the linear viscoelastic region. Dynamic frequency sweep tests were carried out at $28^{\circ} \mathrm{C}$ to observe the storage $\left(G^{\prime}\right)$ and loss modulus $(G$ ') as a function of a wide range of angular frequencies $\left(\omega: 0.1-100 \mathrm{rad}_{\mathrm{s}}^{-1}\right)$. Influence of the composition and the difference in fresh and swelled samples of mumio-based hydrogels on both moduli ( $G^{\prime}$ and $\left.G^{\prime \prime}\right)$ and complex viscosity $\left(\eta^{*}\right)$ which is calculated by Eq. 3, was discussed. 


$$
\eta^{*}=\left[\left(\frac{G^{\prime}}{\omega}\right)^{2}+\left(\frac{G^{\prime \prime}}{\omega}\right)^{2}\right]^{\frac{1}{2}}
$$

The viscoelastic properties of these hydrogels were measured for the samples immediately after preparation (designated as fresh samples) and those after subsequent 60 min swelling in physiological solution (designated as swelled samples) of dried ones.

\section{RESULTS AND DISCUSSION}

\section{Moisture Content}

Initial moisture content of hydrogels is one of the important criteria when considering them for biomedical applications especially as dressing cum wound healing purposes. The moisture content of mumio-based medicated hydrogel is depicted in Table 2. It can be seen from the table that all the hydrogels contain quite a good amount of water (approximately 93-96\%). Maximum amount of water is holding by PVA-agar hydrogel than the others with mumio and other ingredients. It seems that the presence of mumio and other components is hampering to hold water.

TABLE 2. Moisture content of mumio-based medicated hydrogel.

\begin{tabular}{lccc}
\hline Sample & Initial weight (g) & Dry weight (g) & Moisture (\%) \\
\hline PVA-agar (control) & 17.622 & 0.701 & 96.0 \\
PVA-agar-mumio & 10.619 & 0.604 & 94.3 \\
PVA-agar-mumio-SB Oil & 12.897 & 0.842 & 93.5 \\
PVA-agar-mumio-MM & 15.065 & 1.032 & 93.1 \\
PVA-agar-mumio-SA & 16.366 & 1.014 & 93.8 \\
\hline
\end{tabular}

\section{Physical Appearance}

A visual image of mumio-based hydrogels is presented in Figure 1. It can be seen from the figure that PVA-agar hydrogel is white, transparent and soft polymeric gel like material whereas mumio-based medicated hydrogel is brownish, soft but not transparent because of the presence of mumio which is dark brown in colour. One of the images of mumio-based hydrogels is presented here as the rest of the hydrogels are similar in physical appearance. 

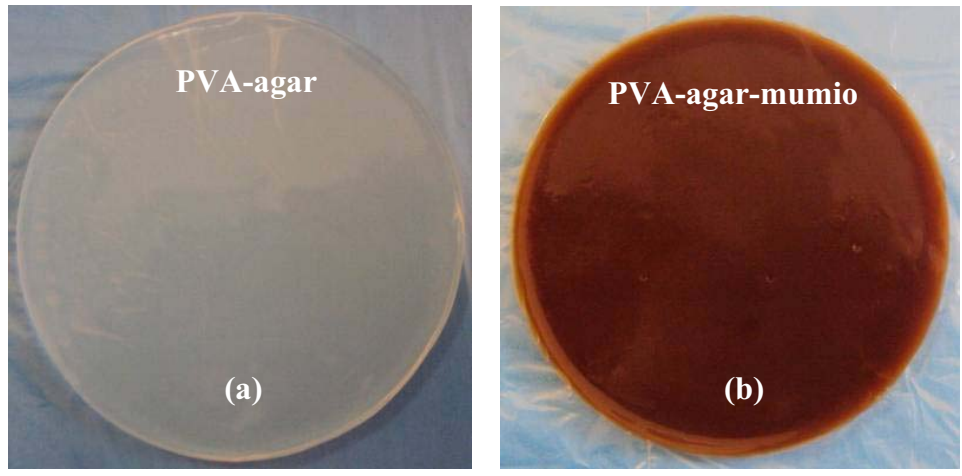

FIGURE 1. Visual image of mumio-based medicated hydrogel: (a) without mumio and (b) with mumio (as an example: PVA-agar-mumio).

\section{Swelling}

Swelling behaviour of mumio-based medicated hydrogels is presented in Figure 2, which shows that the variation in liquid absorption capacity depends on their composition. Though the time required to reach equilibrium swelling state varies from hydrogel to hydrogel (on the basis of hydrogel compositions) but all hydrogels reached in equilibrium condition at the same time i.e. after around $5 \mathrm{~h}$. It can be seen from the figure that hydrogel (PVA-agar) without a healing agent displays significantly higher swelling properties than other mumio-based hydrogels. The lowest degree of swelling is observed in PVA-agar-mumio-MM hydrogel. It can be explained as that combination of mumio and milk may create more cross links into the hydrogel, which causes lowering in swelling percentage.

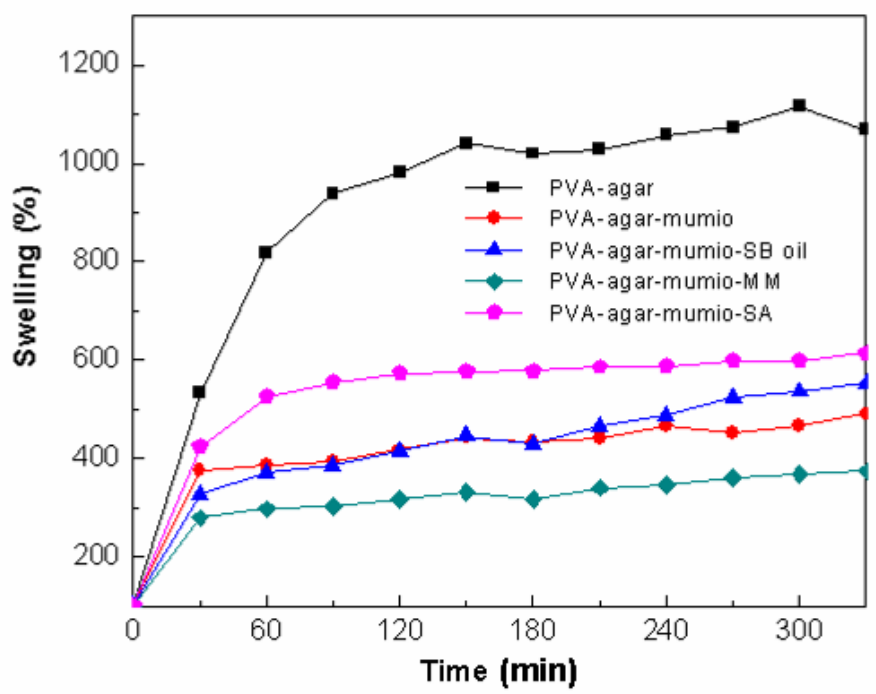

FIGURE 2. Swelling of dry hydrogels at $37^{\circ} \mathrm{C}$ in physiological solution. 


\section{Viscoelastic Properties}

The frequency sweep curve gives a good rheological description of how the product will behave during storage and application. It is considered that the dynamic viscoelasticity of a polymer liquid at low angular frequency $(\omega)$ region is considerably sensitive to structure changes or to the formation of a network structure. To assay the effectivity of angular frequency $\left(0.1-100 \mathrm{rad}_{\mathrm{s}} \mathrm{s}^{-1}\right)$ at a constant strain $(1 \%)$ on viscoelastic properties of hydrogel (i.e. PVA-agar crosslinked gel with and without mumio) was investigated for fresh and swelled samples as shown in Figures 3, 4. Swelled samples were investigated to assess how the swelling affects the viscoelastic behaviour of the gel structure. The gel structure was analysed on the basis of their internal morphology and depicted in Figure 5.

Figure 3 shows the effect of angular frequency $(\omega)$ on storage modulus $\left(G^{\prime}\right)$ and loss modulus ( $\left.G^{\prime \prime}\right)$ for mumio-based hydrogels (fresh and swelled). It can be seen from the figure that in each and every sample, $G$ ' is significantly higher value than $G$ ", and all the samples with varying healing agents were similar in $G$ ' vs. $\omega$ and $G$ " vs. $\omega$ curve patterns. This means that all hydrogels show higher elastic property rather than viscous property. There is no significant change in the values of $G^{\prime}$ and $G$ " on the application of different $\omega$ over the range tested, showing more or less similar to rubbery plateau behaviour. These results mean that the hydrogels are well crosslinked, and then $\omega$ can not influence much to change a largely elastic behaviour with a comparatively small viscous dissipation in energy. This property is very much necessary for hydrogels from the application point of view. Consequently, the $G$ " plots in the measured frequency range were lower than the $G^{\prime}$ plots. Varying the composition of hydrogels and thus also crosslinking change the functionality of base PVA-agar hydrogel that alter the frequency sweep tests results. When compared to PVA-agar hydrogel, the highest elastic property occurred for PVA-agar-mumio-SA blends. Incorporation of mare's milk into the hydrogels also increases the elasticity. SB oil in the blend decreases the $G$ ' values due to intermolecular and intramolecular interactions that can alter the physical state of the hydrogel.
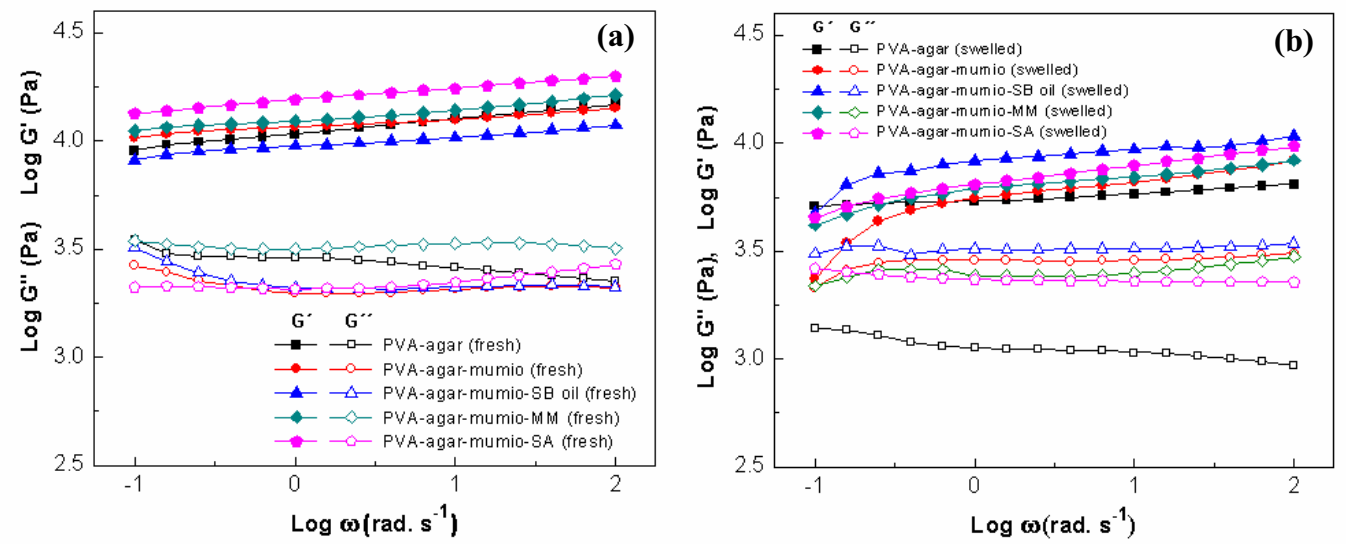

FIGURE 3. Effect of angular frequency $(\omega)$ on storage modulus $\left(G^{\prime}\right)$ and loss modulus $\left(G^{\prime \prime}\right)$ for (a) fresh and (b) swelled mumio-based hydrogels with different healing agents. 
In comparison with the fresh samples, lower viscoelastic behaviour was observed for the samples swelled in water for 60 min except of PVA-agar-mumio-SB oil sample at which elasticity remained the same value and viscosity even increased. The largest swelling effect on rheological properties change, especially decrease of viscosity, was found for a base PVA-agar hydrogel. The hydrogel of PVA-agar-mumio-SA shows best rheological properties with high elasticity.

Complex viscosity $\left(\eta^{*}\right)$ in Figure 4 represents how the water content and addition of healing agent influence on flow behaviour (fluidity) of the hydrogels. It is found from the figure that all the hydrogels show pseudo-plastic behaviour over whole range of angular frequency and follow the "power law" model, and also the other components (healing agents) in the hydrogels influence on the resulting $\eta^{*}$. The influence of $G$ ' on $\eta^{*}$ is predominant than $G$ " for all the measured samples as shown in Figure 3. In comparison to other mumio-based hydrogels, the highest $G^{\prime}$ and $\eta^{*}$ occurred for PVA-agar-mumio-SA blends.

In comparison with fresh samples, lower $\eta^{*}$ was obtained in 60 min swelled samples which contain higher amount of water than fresh ones except of PVA-agar-mumio-SB oil sample at which $\eta^{*}$ remains the same value with very small difference in the lower frequency region.
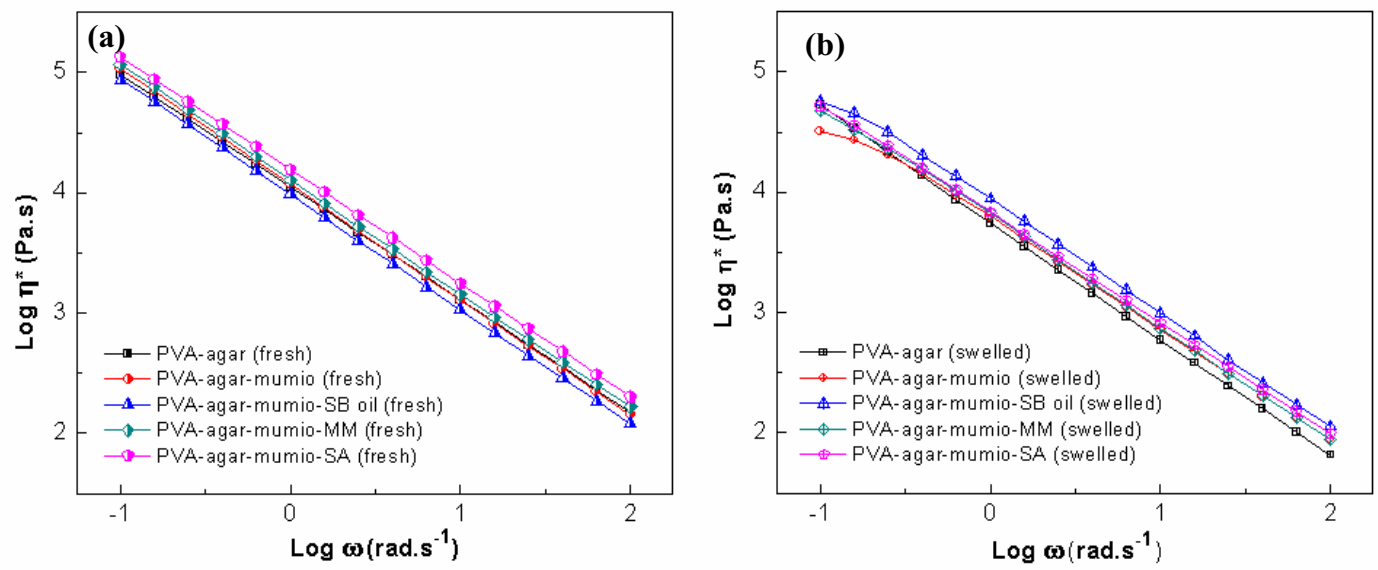

FIGURE 4. Effect of angular frequency $(\omega)$ on complex viscosity $\left(\eta^{*}\right)$ for (a) fresh and (b) swelled PVA-Agar based hydrogels with different healing agents.

\section{Morphology}

The internal gel structure of mumio-based hydrogels with and without mumio was investigated before and after rheological study (viscoelastic measurement). It can be seen from the images shown in Figure 5 that there is significant difference in gel structure before and after measurement at fixed strain (1\%) and after maximum angular frequency of $100 \mathrm{rad} . \mathrm{s}^{-1}$.

It can be seen from the images that during rheological measurement, strain and angular frequency had an effect on the morphology of the hydrogel samples. SEM images reveal that their internal structure change significantly after rheological measurement. On the other hand, among all the hydrogels, PVA-agar-mumio-SA 
shows better porous three-dimensional network (before and after rheological measurement) in comparison to others, which is a very important characteristic of a hydrogel.

\section{Before rheological measurement (a)}
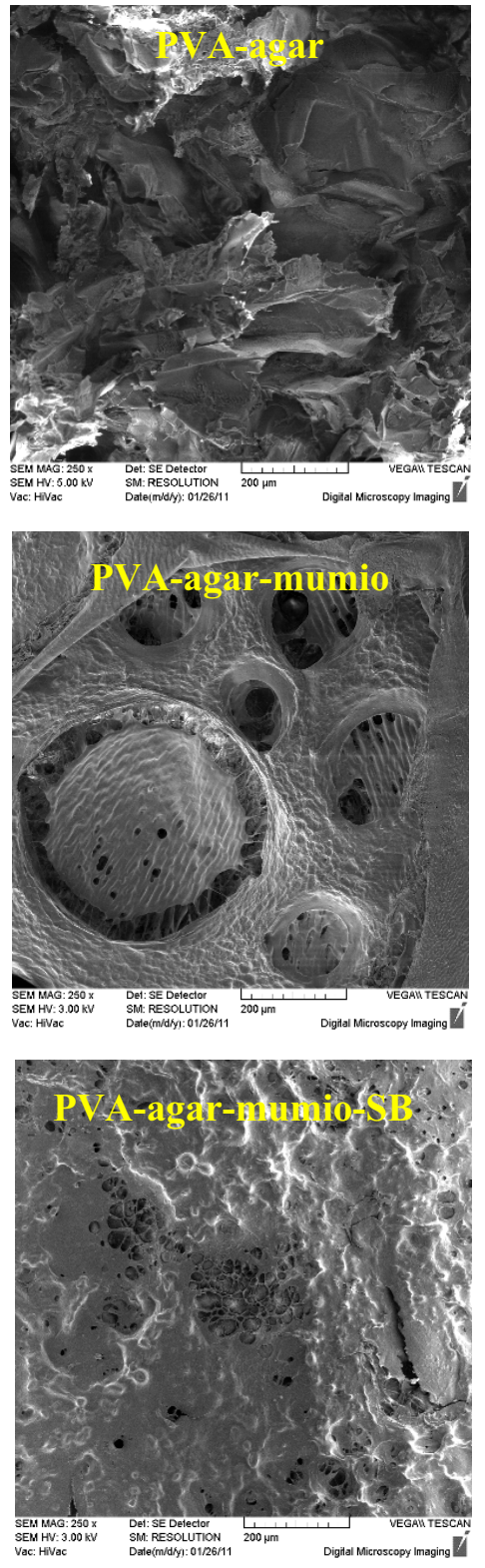

After rheological measurement (b)
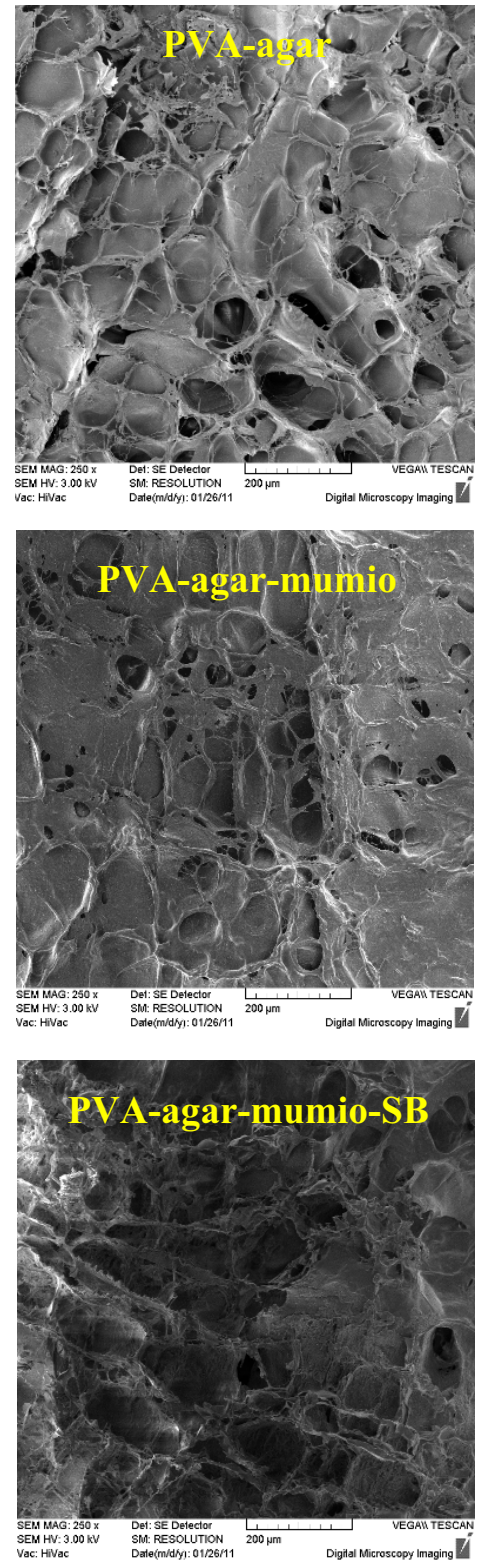


\section{Before rheological measurement (a)}
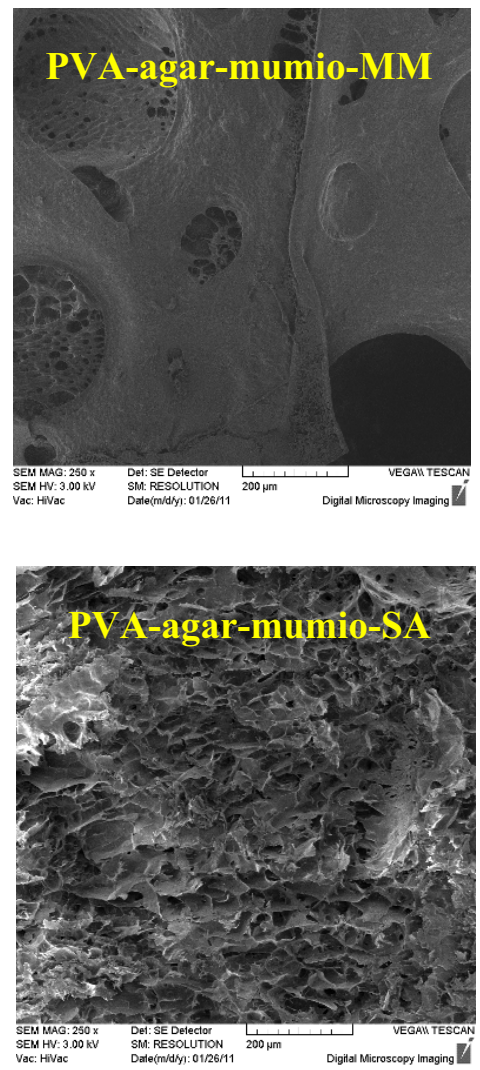

After rheological measurement (b)
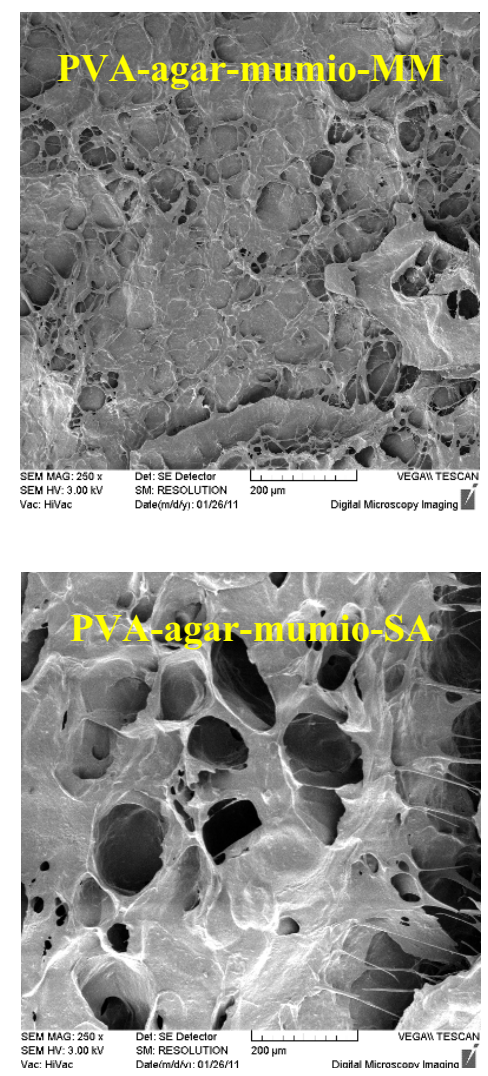

FIGURE 5. SEM image of freeze dried mumio-based hydrogels (a) before and (b) after rheological measurement.

\section{CONCLUSION}

Based on the above observations, we can conclude that interaction between all the components has been occurred adequately. Finally, the morphology of the blend hydrogels showed homogeneous surfaces and confirmed the interaction between the functional groups of the blend components. The swelling study of hydrogels in physiological solution displays remarkable liquid absorption property. Since $G$ ' was always greater than $G$ ", mumio-based hydrogels are mainly elastic materials (negligible viscous effects). In comparison with fresh samples lower viscoelasticity was obtained in swelled samples. According to the obtained results (visoelastic property as well as morphology) of mumio-based medicated hydrogels, hydrogel with SA seems to be the best substance that presents the best attributes for a topical formulation, i.e., more elastic behaviour, higher viscosity as well exhibits good crosslinking net work. 


\section{ACKNOWLEDGMENTS}

The authors are thankful to the support of Operational Program Research and Development for Innovations co-funded by the European Regional Development Fund (ERDF) and national budget of Czech Republic, within the framework of project Centre of Polymer Systems (reg. number: CZ.1.05/2.1.00/03.0111) and secondly to the Ministry of Industry and Trade of the Czech Republic: Project number 2A-1TP1/126.

\section{REFERENCES}

1. E. H. Schacht, J. Phys. Conf. Ser. 3, 22 (2004).

2. F. Lenzi, A. Sannino, A. Borriello, F. Porro, D. Capitani, and G. Mensitieri, Polymer 44, 1577-1588 (2003).

3. Y. M. Lim, S. J. An, H. K. Kim, Y. H. Kim, M. H. Youn, H. J. Gwon, J. Shin, and Y. C. Nho, Radiat. Phys. Chem. 78, 441-444 (2009).

4. M. P. Bagnas (2005) Study of water diffusion through hydrogels. Published on Graduate College at Illinois (http://www.grad.uiuc.edu).

5. A. V. Mondino, M. E. Gonzalez, G. R. Romero, and E. E. Smolko, Radiat. Phys. Chem. 55, 723-726 (1999).

6. J. G. Lyons, L. M. Geever, M. J. D. Nugent, J. E. Kennedy and C. L. Higginbotham, J. Mech. Behavior of Biomed. Mat. 2, 485-493 (2009).

7. L. Varshney, Nucl. Instr. \& Meth. in Phys. Res. B 255, 343-349 (2007).

8. A. Garedew, M. Fiest, E. Schmolz and I. Lamprecht, Thermochimica Acta 417, 301-309 (2004).

9. N. Roy, N. Saha, T. Kitano and P. Saha, AIP Conference Proceedings 1152, 210-216 (2009).

10. L. Weng, X. Chen and W. Chen, Biomacromolecules 8, 1109-1115 (2007).

11. S. Mourtas, M. Haikou, M. Theodoropoulou, C. Tsakiroglou and S. G. Antimisiaris, J. Coll. Interface Sci. 317, 611-619 (2008).

12. T. M. Aminabhavi, S. A. Agnihotri and B. V. K. Naidu, J. Appl. Polym. Sci. 94, 2057-2064 (2004).

13. N. Roy, N. Saha, T. Kitano and P. Saha, Soft Materials 8, 130-148 (2010).

14. K. R. Park and Y. C. Nho, Radiat. Phys. Chem. 67, 361-365 (2003). 
Copyright of AIP Conference Proceedings is the property of American Institute of Physics and its content may not be copied or emailed to multiple sites or posted to a listserv without the copyright holder's express written permission. However, users may print, download, or email articles for individual use. 